Research Article

\title{
Baseline Demographic and Clinical Characteristics of Patients with Adrenal Incidentaloma from a Single Center in China: A Survey
}

\author{
Lele Li, ${ }^{1,2}$ Guoqing Yang, ${ }^{1,2}$ Ling Zhao, ${ }^{1,2}$ Jingtao Dou, ${ }^{1,2}$ Weijun Gu, ${ }^{1,2}$ Zhaohui Lv, ${ }^{1,2}$ \\ Juming $\mathrm{Lu}^{1,2}$ and Yiming $\mathrm{Mu}^{1,2}$ \\ ${ }^{1}$ Department of Endocrinology, Chinese PLA General Hospital, Beijing 100853, China \\ ${ }^{2}$ Chinese PLA Key Laboratory of Endocrinology and Metabolism, Beijing 100853, China \\ Correspondence should be addressed to Jingtao Dou; jingtaodou@sohu.com
}

Received 20 March 2017; Revised 14 June 2017; Accepted 19 June 2017; Published 7 August 2017

Academic Editor: Andrea G. Lania

Copyright (C) 2017 Lele Li et al. This is an open access article distributed under the Creative Commons Attribution License, which permits unrestricted use, distribution, and reproduction in any medium, provided the original work is properly cited.

\begin{abstract}
Aim. To investigate the clinical and endocrinological characteristics of patients with adrenal incidentaloma (AI). Materials and Methods. This retrospective study enrolled 1941 AI patients hospitalized at the Department of Endocrinology, Chinese PLA General Hospital, Beijing, China, between January 1997 and December 2016. The patient gender, age at visits, imaging features, functional status, and histological results were analyzed. Results. Of the 1941 patients, 984 (50.70\%) were men. The median age was 52 years (interquartile range: 44-69 years). 140 cases had bilateral AI. Endocrine evaluation showed that $1411(72.69 \%)$ patients had nonfunctional tumor, 152 (7.83\%) had subclinical Cushing syndrome (SCS), and 82 $(4.33 \%)$ had primary hyperaldosteronism. A total of 925 patients underwent operation for removal of 496 cortical adenomas (53.62\%), 15 adrenal cortical carcinomas (1.62\%), and 172 pheochromocytomas (18.59\%). The bilateral group had a higher proportion of SCS $(18.57 \%$ versus $7.10 \%, P<0.001, P=0.006)$. A mass size of $46 \mathrm{~mm}$ was of great value in distinguishing malignant tumors from the benign tumors, with sensitivity of $88.2 \%$ and specificity of $95.5 \%$. Conclusions We reported the baseline demographic and clinical characteristics of patients with AI in a large series from a single center in China.
\end{abstract}

\section{Introduction}

An adrenal incidentaloma (AI) is a previously unsuspected adrenal mass discovered on an imaging study performed for an unrelated reason. Increased use of computed tomography (CT), ultrasonography, and magnetic resonance imaging (MRI) has led to its frequent discovery. Current estimates of the prevalence of AI ranges in various studies: $2.3 \%$ at autopsy, $4 \%$ in retrospective CT series, and 5.8\% in oncological studies; its prevalence reportedly increases with aging [1-3]. As AI is increasingly recognized in current medical practice, such masses raise challenging questions for both physicians and their patients and represent one of the leading reasons for endocrinological consultation [4].
The term AI is not a single entity, rather it is an "umbrella" definition comprising a spectrum of different pathological entities that share the same path of discovery [5]. Only malignant or hyperfunctional AI (excess production of cortisol, aldosterone, and catacholamines) needs active treatment; thus, it is mandatory to identify such patients. A number of clinical series regarding AI have been reported in the literature [6-11]; however, high heterogeneity among data sources has been noted, and there is no international consensus regarding the management of AI. Moreover, studies of $\mathrm{AI}$ in the Chinese population are insufficient. Therefore, we conducted a retrospective analysis of the clinical and endocrinological characteristics of patients with $\mathrm{AI}$ at a tertiary referral hospital in China to provide greater insight into their work-up and management. 


\section{Materials and Methods}

This retrospective study was conducted at the Department of Endocrinology, Chinese PLA General Hospital, Beijing, China. The Chinese PLA General Hospital Ethics Committee specifically approved this study, and written informed consent was obtained from all patients.

According to the definition of AI, we excluded patients with the following: (1) clinical symptoms relevant to the adrenal gland; (2) abnormal biochemical results, that is, severe paroxysmal hypertension, hypokalemia, and clinical signs of hypercortisolism or hyperandrogenism, indicating abnormal adrenal function prior to initial radiological investigation; or (3) previous history of malignancies. However, patients with adrenal metastasis as the initial discovery of a malignancy were included. Patient information was obtained retrospectively from their medical records by use of a specifically tailored questionnaire. All completed questionnaires were individually checked for inconsistencies before statistical analysis, and double inclusion of the same patient was avoided. Finally, 1941 eligible patients hospitalized between January 2006 and December 2016 were enrolled.

After history and physical examination, all patients underwent biochemical evaluation to assess their functional status. Biochemical evaluation consisted of measurements of patients' $24 \mathrm{~h}$ urine-free cortisol level (2 times); serum cortisol and plasma ACTH levels at 0:00, 8:00, and 16:00, followed by overnight dexamethasone test (DST, $1 \mathrm{mg}$ dexamethasone given at 23:00); and measurement of serum cortisol and plasma ACTH levels at 8:00 the next morning $(<50 \mathrm{nmol} / \mathrm{L}$ was considered normal). Patients with postDST cortisol levels $\geq 50 \mathrm{nmol} / \mathrm{L}$ underwent additional tests, specifically the low-dose DST (LDDST, $4 \mathrm{mg} / 48 \mathrm{~h}$; cutoff level, $50 \mathrm{nmol} / \mathrm{L})$. Patients with post-LDDST cortisol levels $\geq 50 \mathrm{nmol} / \mathrm{L}$ were diagnosed as having subclinical Cushing syndrome (SCS). Patients with an aldosteronerennin ratio $(A R R>20)$ underwent any of the following three confirmatory tests to definitively confirm or exclude PA: saline infusion test, captopril challenge test, and postural stimulation test. Pheochromocytoma was diagnosed on the basis of the concentrations of catecholamines and metanephrines in two $24 \mathrm{~h}$ urine samples and adrenal CT or MRI scan before operation and confirmed or corrected according to the histological analysis. The diagnosis of $\mathrm{CAH}$ was based on clinical history, current clinical status, and hormonal criteria (mainly 17-OHP) and confirmed by CYP21A2 gene mutational analysis. After ruling out the above status, a diagnosis of nonfunctional adrenal tumor was established.

All the hormones in the study were analyzed by chemiluminescence immunoassay. Cortisol and ACTH levels were analyzed by chemiluminescence immunoassay. ACTH was detected using an Immulite 2000 Analyzer (Siemens Healthcare Diagnostics Inc., LA, USA). Cortisol was measured with an ADVIA Centaur Analyzer (Siemens Healthcare Diagnostics, Tarrytown, NY, USA). The CT imaging technique was not standardized because of the different hospitals that patients initially visited, and only CT-estimated mass

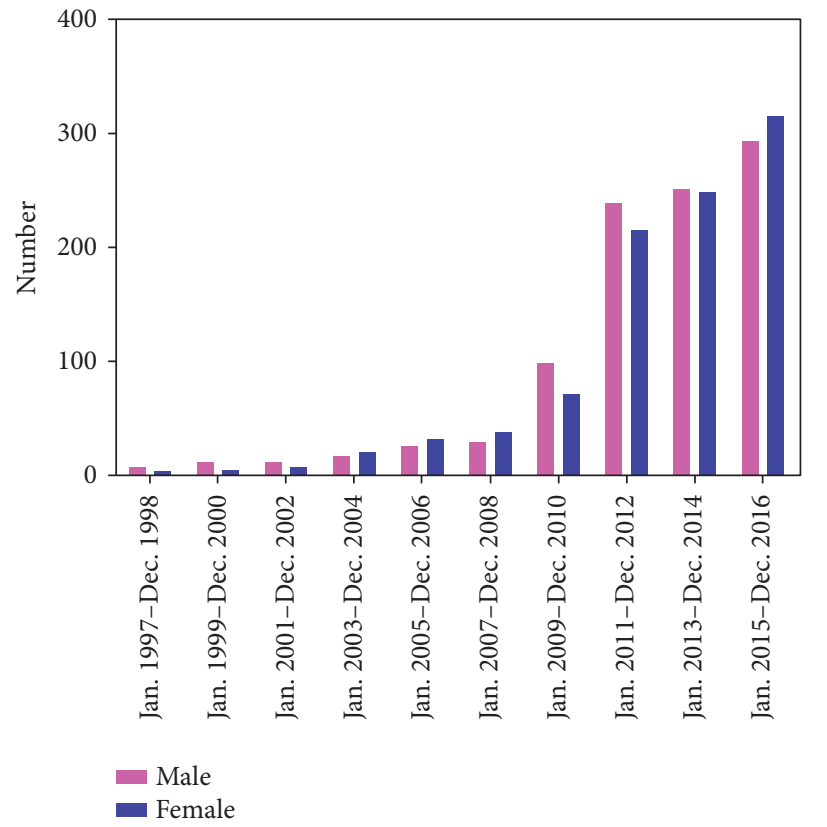

FIGURE 1: The distribution of patients according to the year.

location and size were considered for statistical analysis. Histological diagnosis was reported according to the original description, with a central review of pathological specimens.

Statistical analysis was performed using SPSS Software (Version 17.0). Values are expressed as median (interquartile range) or as numbers with percentage. Categorical data such as gender and clinical/radiologic features were compared using $\chi^{2}$ test. Group data with a normal distribution were compared using a $t$-test. A two-sided $P$ value $<0.05$ was considered to indicate statistical significance.

\section{Results}

3.1. General Characteristics of Patients with AI. Between January 2006 and December 2016, 1941 patients eligible were enrolled. The distribution of patients according to the year is shown in Figure 1, which shows that the number of cases gradually increased each year. The demographic characteristics are presented in Table 1. Of the 1941 patients, 984 (50.70\%) were men. The median age of patients was 52 years (interquartile range, 44-69 years), and the distribution of patients according to age is illustrated in Figure 2: the highest proportion of AI was in patients aged 50-60 years. Overall, unilateral tumors were detected on the left side in 865 (46.93\%) cases and on the right side in 838 (45.47\%); the remaining 140 cases had bilateral AI. Hypertension was observed in 1045 (53.9\%) patients, diabetes in 257 (13.3\%) patients, dyslipidemia in $576(29.8 \%)$ patients, fatty liver in 786 (45.04\%, 1742 patients with data available) patients, and obesity (body mass index [BMI] $>28 \mathrm{~kg} / \mathrm{m}^{2}$ ) in 514 (26.48\%) patients. Routine medical checkup was the main method (42.14\%) for detection of clinical onset leading to disease discovery. The predominant complaint included low back pain $(7.41 \%)$ and abdominal pain $(13.80 \%)$. 
TABLE 1: Baseline demographic and general characteristics of patients with adrenal incidentaloma.

\begin{tabular}{lc}
\hline Characteristic & Value \\
\hline Gender, male/female, $N$ & $984 / 957$ \\
Age, median (IQR), $y$ & $52(44,59)$ \\
BMI, median (IQR), $\mathrm{kg} / \mathrm{m}^{2}$ & $25.63(23.19,28.06)$ \\
$\leq 24 \mathrm{~kg} / \mathrm{m}^{2}, N(\%)$ & $578(29.78)$ \\
$>24, \leq 28 \mathrm{~kg} / \mathrm{m}^{2}, N(\%)$ & $849(43.74)$ \\
$>28 \mathrm{~kg} / \mathrm{m}^{2}, N(\%)$ & $514(26.48)$ \\
Location, $N(\%)^{\mathrm{a}}$ & \\
Left & $865(46.93)$ \\
Right & $838(45.47)$ \\
Both & $140(7.60)$ \\
Mass size, median (IQR), mm ${ }^{\mathrm{b}}$ & $23(16,36)$ \\
Surgery, $N$ (\%) & $925(47.65)$ \\
Concomitant disease, $N(\%)$ & \\
Diabetes mellitus & $257(13.3)$ \\
Hypertension & $1045(53.9)$ \\
Dyslipidemia & $576(29.8)$ \\
Fatty liver & $786(45.04)$ \\
Reasons for initial imaging, $N(\%)$ & \\
Routine medical checkup & $818(42.14)$ \\
Low back pain & $144(7.41)$ \\
Abdominal pain & $268(13.80)$ \\
Urinary tract disease & $81(41.73)$ \\
Respiratory system disease & $133(6.85)$ \\
Hepatobiliary disease & $817(21.43)$ \\
Others & \\
\hline & \\
\hline &
\end{tabular}

${ }^{a} 1843$ patients with data available. ${ }^{b}$ Widest diameter of the largest tumor among 1736 patients with data available. ${ }^{c} 1742$ patients with data available. IQR: interquartile range; BMI: body mass index.

3.2. Functional Status and Histological Results of Patients with AI. Endocrinological evaluation showed that 1411 (72.69\%) patients had nonfunctional tumor, $152(7.83 \%)$ had subclinical Cushing syndrome (SCS), 82 (4.33\%) had primary hyperaldosteronism, and 227 (11.69\%) had pheochromocytomas. Of the 561 patients with available $17-\mathrm{OH}-$ progesterone (17-OHP) data, 4 were confirmed to have congenital adrenal hyperplasia $(\mathrm{CAH})$. A total of 925 patients underwent operation for removal of 496 cortical adenomas (53.62\%), 15 adrenal cortical carcinomas (1.62\%), 172 pheochromocytomas (18.59\%), 45 paragangliomas/ganglioneuromas (4.86\%), 54 cysts (5.85\%), and 41 myelolipomas (4.43\%). Table 2 presents the detailed functional status and histological results of the patients.

3.3. Clinical Characteristics and Functional Status of Patients with Bilateral and Unilateral AI. As shown in Table 3, patients with bilateral and unilateral AI did not differ in terms of median age and BMI; however, the gender difference was statistically significant between the two groups $(P=0.001)$. The bilateral AI group had a higher proportion of male patients than the unilateral AI group. The

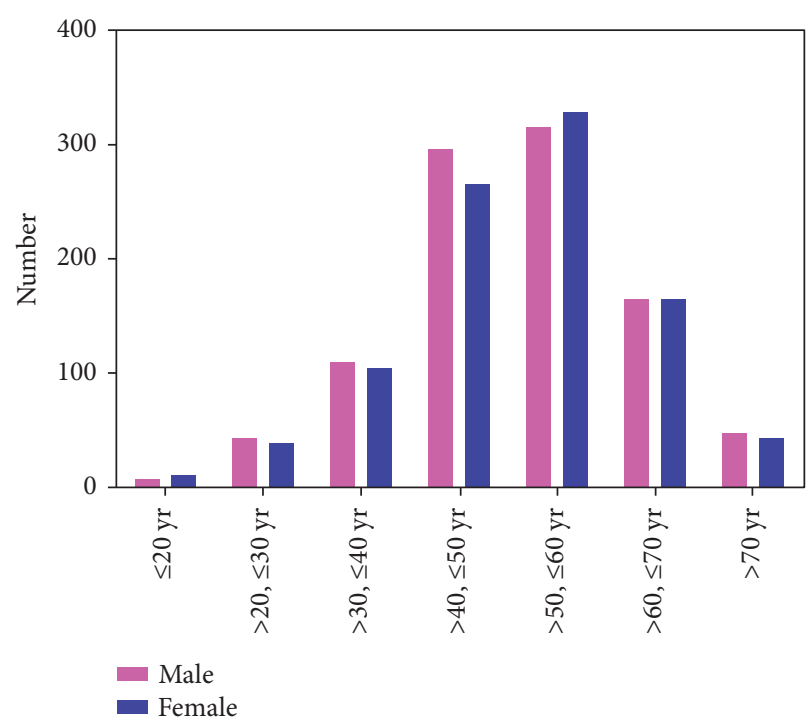

FIgURE 2: The distribution of patients according to age.

patients' functional status showed that the bilateral group had a higher proportion of patients with SCS (18.57\% versus $7.10 \%, P<0.001)$ and a lower proportion of patients with pheochromocytoma ( $4.29 \%$ versus $11.92 \%, P=0.006)$ than the unilateral group. The proportion of nonfunctional tumor and PA showed no differences between the two groups.

3.4. Clinical Characteristics and Tumor Types by Size. Of the 925 operated patients, 828 had data available on the mass size. On the basis of the widest diameter of the largest tumor, these cases were classified into the following groups: $\leq 2 \mathrm{~cm}$ $(N=159), 2-4 \mathrm{~cm}(N=828), 4-6 \mathrm{~cm}(N=398)$, and $>6 \mathrm{~cm}$ $(N=127)$. The clinical characteristics and tumor types are presented in Table 4. The highest proportion of SCS, PA, and pheochromocytomas was observed in the $2-4 \mathrm{~cm}$, $\leq 2 \mathrm{~cm}$, and $4-6 \mathrm{~cm}$ groups, respectively. In the $>6 \mathrm{~cm}$ group, the proportion of malignancy increased sharply. The proportion of each tumor type according to mass size is presented in Figure 3. Receiver-operating characteristic (ROC) analysis was performed to evaluate the diagnostic value of mass size for distinguishing between malignant and benign tumors. The area under the curve (AUC) was 0.946 (95\% confidence interval: $0.892-1$ ), and the optimal cutoff value was $46 \mathrm{~mm}$, with a sensitivity of $88.2 \%$ and specificity of $95.5 \%$, shown in Figure 4.

\section{Discussion}

To the best of our knowledge, this is the largest clinical series of AI reported from a single center in the literature. AIs are being detected with an increasing frequency due to the widespread increase in cross-sectional imaging and is gradually emerging as a common clinical problem. Our data show that 1562 (80.47\%) patients hospitalized between January 2011 and December 2016 had AI, indicating an increasing rate of detection and greater awareness of AI.

Age distribution of our series of patients was wide, and the median age at visits was 52 years, which is in line with 
TABLE 2: Functional status and histological results of patients with adrenal incidentaloma.

\begin{tabular}{lccc}
\hline Functional status & $N(\%)$ & Histological result & $N(\%)$ \\
\hline Nonfunctional tumor & $1411(72.69)$ & Adrenal adenoma & $496(53.62)$ \\
Subclinical Cushing syndrome & $152(7.83)$ & Pheochromocytoma & $172(18.59)$ \\
Primary hyperaldosteronism & $82(4.22)$ & Paragangliomas/ganglioneuromas & $45(4.86)$ \\
Pheochromocytoma & $227(11.69)$ & Adrenal cortical carcinoma & $15(1.62)$ \\
Adrenal cortical carcinoma & $25(1.29)$ & Cyst & $54(5.84)$ \\
Congenital adrenal hyperplasia & $4^{\mathrm{a}}$ & Myelolipoma & $41(4.43)$ \\
Unknown functional status & $40(2.06)$ & Schwannoma & $10(1.08)$ \\
& & Hyperplasia & $15(1.62)$ \\
& & Metastatic carcinoma & Others \\
\end{tabular}

${ }^{\mathrm{a}} 561$ patients with 17 -OHP data available. ${ }^{\mathrm{b}}$ Including hematoma, teratoma, sarcoma, hemangioma, lymphangioma, and lymphoma, 20 patients operated without histological results.

TABle 3: Clinical characteristics and functional status of patients with bilateral and unilateral adrenal incidentaloma.

\begin{tabular}{|c|c|c|c|}
\hline & $\begin{array}{c}\text { Unilateral } \\
(N=1703)\end{array}$ & $\begin{array}{c}\text { Bilateral } \\
(N=140)\end{array}$ & $P$ value \\
\hline Gender, male/female, $N$ & $852 / 851$ & $86 / 54$ & 0.010 \\
\hline Age, median (IQR), y & $52(44,59)$ & $55(49,62)$ & 0.484 \\
\hline BMI, median (IQR), $\mathrm{kg} / \mathrm{m}^{2}$ & $25.56(23.14,28.04)$ & $26.64(24.39,28.86)$ & 0.283 \\
\hline Mass size, median (IQR), mm & $22(15,36)$ & $20(12,29)$ & 0.041 \\
\hline Surgery, $N(\%)$ & $828(48.62)$ & $46(32.86)$ & $<0.001$ \\
\hline \multicolumn{4}{|l|}{ Functional status, $N(\%)$} \\
\hline Nonfunctional tumor & $1254(73.63)$ & $97(69.29)$ & 0.263 \\
\hline Subclinical Cushing syndrome & $121(7.10)$ & $26(18.57)$ & $<0.001$ \\
\hline Primary hyperaldosteronism & $73(4.29)$ & $5(3.57)$ & 0.686 \\
\hline Pheochromocytoma & $203(11.92)$ & $6(4.29)$ & 0.006 \\
\hline Adrenal cortical carcinoma & $24(1.41)$ & 0 & - \\
\hline Congenital adrenal hyperplasia & 1 & 3 & - \\
\hline Unknown functional status & 27 & 3 & - \\
\hline
\end{tabular}

IQR: interquartile range.

TABLE 4: Clinical characteristics and tumor types categorized by size in 828 operated patients.

\begin{tabular}{|c|c|c|c|c|}
\hline & $\begin{array}{c}\leq 2 \mathrm{~cm} \\
(N=159)\end{array}$ & $\begin{array}{l}>2, \leq 4 \mathrm{~cm} \\
(N=398)\end{array}$ & $\begin{array}{l}>4, \leq 6 \mathrm{~cm} \\
(N=144)\end{array}$ & $\begin{array}{c}>6 \mathrm{~cm} \\
(N=127)\end{array}$ \\
\hline Gender, male/female, $N$ & $79 / 80$ & $181 / 217$ & $80 / 64$ & $66 / 61$ \\
\hline Age, median (IQR), y & $51(45,59)$ & $51(44,58)$ & $47(37,56)$ & $44(34,53)$ \\
\hline BMI, median (IQR), $\mathrm{kg} / \mathrm{m}^{2}$ & $25.65(23.38,27.94)$ & $25.85(23.12,28.40)$ & $24.26(22.06,26.58)$ & $24.42(21.19,26.64)$ \\
\hline \multicolumn{5}{|l|}{ Type of tumor, $N(\%)$} \\
\hline Nonfunctional adenoma & $84(52.83)$ & $189(47.49)$ & $21(14.58)$ & $10(7.87)$ \\
\hline Subclinical Cushing syndrome & $19(11.95)$ & $70(17.59)$ & $11(7.64)$ & $2(1.57)$ \\
\hline Primary hyperaldosteronism & $29(18.24)$ & $12(3.02)$ & $0(0)$ & $0(0)$ \\
\hline Pheochromocytoma & $7(4.40)$ & $57(14.32)$ & $59(40.97)$ & $43(33.85)$ \\
\hline Paragangliomas/ganglioneuromas & $2(1.26)$ & $14(3.52)$ & $11(7.64)$ & $13(10.24)$ \\
\hline Myelolipoma & $2(1.26)$ & $12(7.55)$ & $12(8.33)$ & $12(9.45)$ \\
\hline Cyst & $5(3.14)$ & $25(6.28)$ & $11(7.64)$ & $4(3.15)$ \\
\hline Adrenal cortical carcinoma & $0(0)$ & $1(0.25)$ & $1(0.69)$ & $12(9.45)$ \\
\hline Other malignant tumors & $1(0.63)$ & $2(0.50)$ & $7(4.86)$ & $13(10.24)$ \\
\hline Others & $10(6.3)$ & $15(3.77)$ & $11(7.64)$ & 19 (14.96) \\
\hline
\end{tabular}




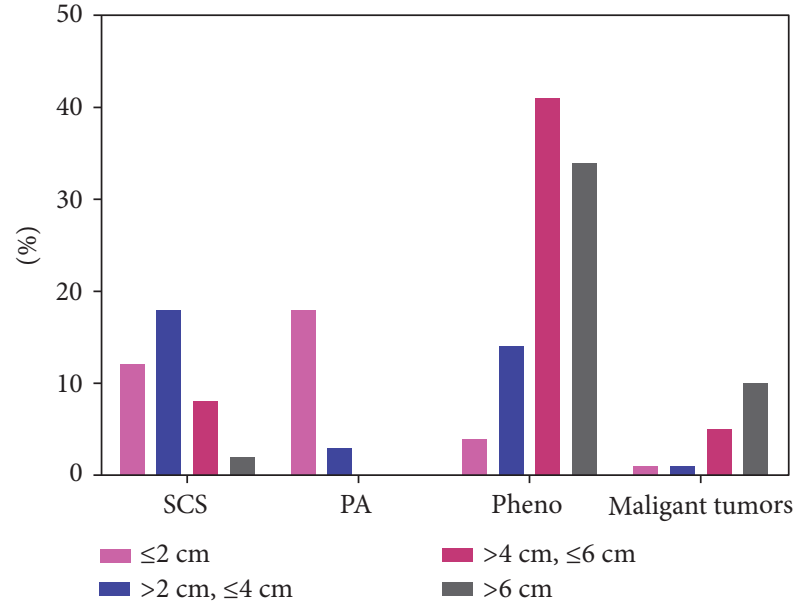

Figure 3: Proportion of each type of tumor according to the size. Malignant tumors include adrenal cortical carcinoma, metastatic carcinoma, lymphoma, and sarcoma. SCS: Subclinical Cushing syndrome; PA: primary hyperaldosteronism; Pheo: pheochromocytoma.

the findings of previous studies $[8,10,12]$. The increasing age of the general population and a trend towards more advanced investigations in the elderly maybe have contributed to the high detection rate in this age group. Alternatively, the high detection rate could be explained by an increased occurrence of cortical nodules with age, as observed in unselected autopsy series $[13,14]$. This finding may represent a compensatory growth in response to the local ischemic damage of arteriosclerotic disease [15]. Mantero et al. [10] and Tabuchi et al. [8] reported a female tendency in their study, but our case series did not report significant gender differences; this could be partly explained by a referral bias, as nonfunctional adrenal adenomas occurred with comparable frequency in men and women in autopsy series $[13,14]$. In the present study, we found that the frequency of AI in both sides was comparable, and this finding is consistent with the recent literature published in 2013 and 2016 [6, 8, 16]; previous studies have shown contrasting results $[10,17]$. These discrepancies might be due to the advances in medical imaging technology in recent years: ultrasonography was the diagnostic technique used in the previous series, and the right adrenal gland was better visualized by ultrasound than the left gland [18]. Therefore, recent advances in CT technology might have improved the detection of small tumors, especially in the left adrenal gland.

Clinically, upon discovery of AIs, two issues arise: functionality and malignancy. Regarding the functionality of masses, we observed that 1411 (72.69\%) patients had nonfunctional tumors. Among the functional tumors, pheochromocytomas (11.69\%) were the most frequently observed in our series, followed by SCS (7.83\%) and PA $(4.22 \%)$. In the literature, the frequency of pheochromocytomas ranged between 1.5 and $23 \%$, whereas that of SCS varied from $1.2 \%$ to $12 \%$ [5]; such a great variability could be attributed to the inclusion criteria and referral pattern

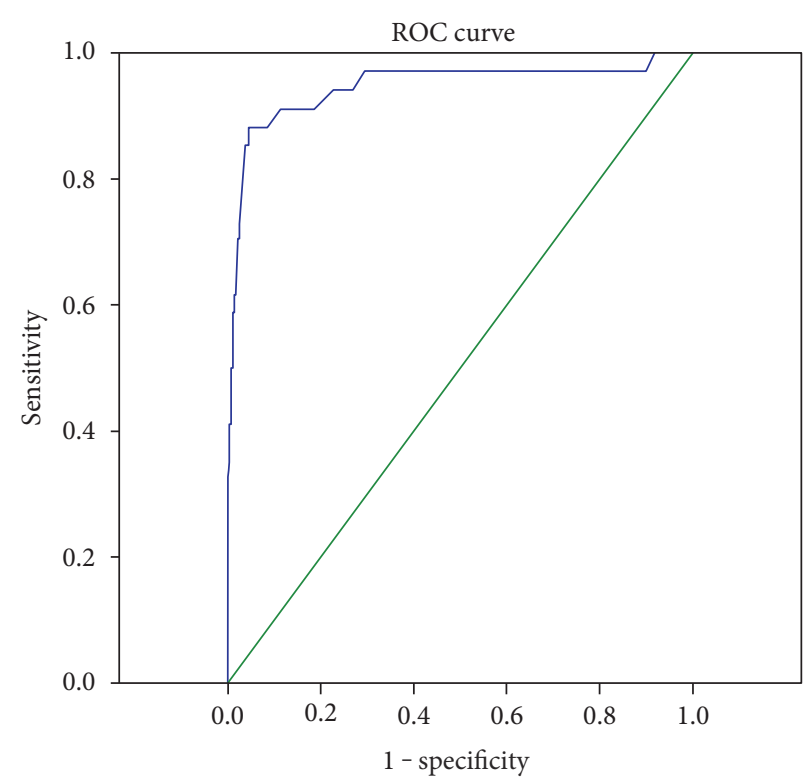

FIgURE 4: Area under the receiver-operating characteristic curves for the diagnosis of malignant and benign tumors.

of various studies. Furthermore, analysis of the hormonal data collected in our series confirmed that an endocrine work-up in patients with AI led to the detection of a remarkable number of subclinical hormone-producing tumors, which further addresses the importance of endocrine evaluation.

Bilateral lesions were detected in $7.6 \%$ of cases in the present series. Compared to patients with unilateral lesions, a higher frequency of SCS and a lower frequency of pheochromocytomas were detected in patients with bilateral lesions. Other series have also reported similar results $[19,20]$. Moreover, previous studies indicated the probability that SCS is positively correlated with mass size [19]; this finding persisted when patients with unilateral AI were analyzed separately from those with bilateral AI [21, 22]. Our series showed that the highest proportion of SCS, PA, and pheochromocytomas were observed in $2 \sim 4 \mathrm{~cm}, \leq 2 \mathrm{~cm}$, and $4 \sim 6 \mathrm{~cm}$ groups, respectively. Figure 5(a) shows the individual size values of different functional tumors. With regard to the precise assessment of SCS caused by bilateral lesions, previous studies assumed that the adrenal mass size in patients with unilateral AI was comparable with that of the largest mass in those with bilateral lesions and that in most patients with bilateral adrenal masses and SCS, one side of the adrenal gland, the larger side, is hypersecreting. All the above evidences support the importance of the mass size in the evaluation of its functional status. Notably, 3 of the 4 patients diagnosed with $\mathrm{CAH}$ initially presented with massive bilateral incidentalomas. In recent years, it has become evident that both homozygous and heterozygous patients with CAH have a high prevalence of AI [23-25]. In addition, although rare, some patients with AI were confirmed to have nonclassical CAH. The diagnosis of $\mathrm{CAH}$ in patients with AI is mandatory, as the patients are at risk for adrenal crises. The latest AI guidelines issued by the European Society of 


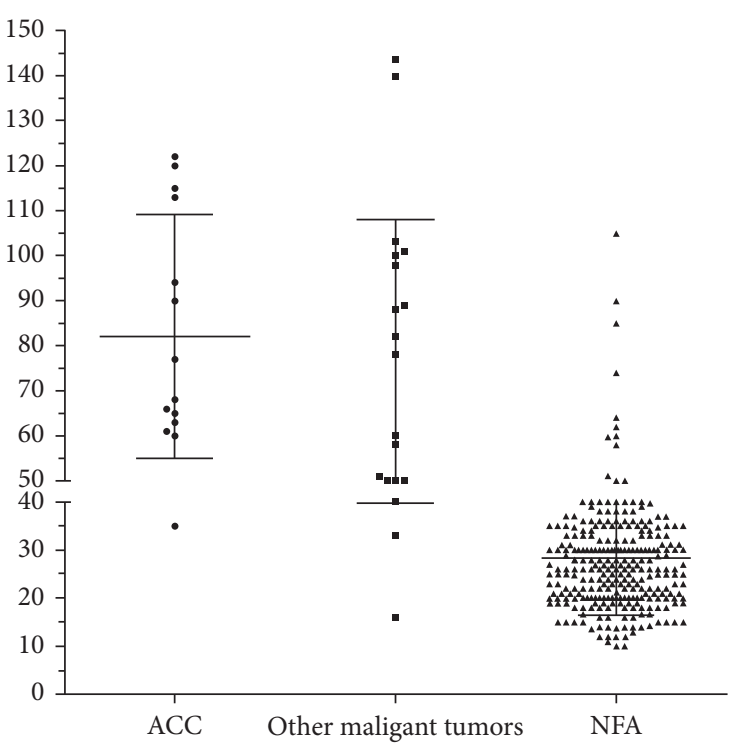

(a)

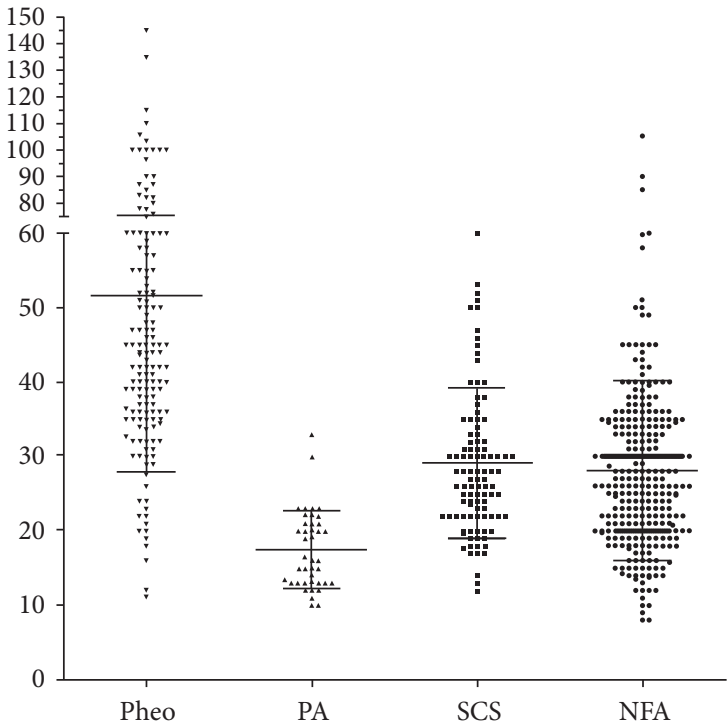

(b)

FIgURE 5: (a) The individual sizes of different functional tumors. (b) The individual sizes of benign and malignant tumors. Other malignant tumors include metastatic carcinoma, lymphoma, and sarcoma. SCS: subclinical Cushing syndrome; PA: primary hyperaldosteronism; Pheo: pheochromocytoma; ACC: adrenal cortical; carcinoma.

Endocrinology and European Network for the Study of Adrenal Tumors have recommended screening of $\mathrm{CAH}$ in patients with bilateral AI [26]. If AI is suspected clinically, assays for cortisol, adrenocorticotropic hormone (ACTH), dehydroepiandrosterone sulfate (DHEAS), and 17-OHP should be performed.

Regarding the malignancy of the masses, 37 of the 828 operated patients were confirmed to have malignant tumors by a final histological analysis. In the $>6 \mathrm{~cm}$ group, the frequency of malignancy increased sharply. The size of individual benign and malignant tumors is shown in Figure 5(b). Overall, cortical adenomas were the smallest lesions, whereas malignant tumors were the largest lesions, despite some overlap. These findings confirm that the risk of malignancy is related to mass size. Previous studies suggest that nearly all lesions measuring $<4 \mathrm{~cm}$ are benign and $<2 \%$ are malignant $[7,27]$. In the present study, ROC analysis was performed to evaluate the diagnostic value of mass size to distinguish between malignant and benign tumors; the results showed an AUC of 0.946 and optimal cutoff value of $46 \mathrm{~mm}$, with a sensitivity of $88.2 \%$ and specificity of $95.5 \%$. In China, overtreatment of nonfunctional AI measuring $<4 \mathrm{~cm}$ is frequent due to a lack of agreement on this entity. Here, we suggest that tumors in this group, which are defined as having a low risk of malignancy by the imaging criteria, are generally not surgically resected. In addition to imaging characteristics, hormonal measurements could aid in the differential diagnosis because a great elevation of adrenal androgen levels, and in particular, DHEAS, could indicate the presence of primary adrenal malignancy. Thus, comprehensive analysis of imaging features and endocrinological results might be of remarkable clinical significance.

There are several limitations to our study that should be discussed. First, this was a retrospective review of cases from a single center. Second, some data were unavailable, which decreased the power of analysis. Finally, there was a lack of clinical and biochemical follow-up of the patients.

\section{Conclusion}

We reported the baseline demographic and clinical characteristics, especially the functionality and malignancy, of patients with $\mathrm{AI}$ in a large series from a single center in China. A total of $72.69 \%$ patients were diagnosed with nonfunctional tumors. Among the functional tumors, pheochromocytomas (11.69\%) were the most frequently observed, followed by SCS $(7.83 \%)$ and PA $(4.22 \%)$. Bilateral AI was detected in $7.6 \%$ of the study population, and a higher proportion of SCS and a lower proportion of pheochromocytomas were seen in these cases as compared to the unilateral $\mathrm{AI}$ cases. Mass size was of great value in distinguishing malignant and benign tumors.

\section{Conflicts of Interest}

The authors declare that they have no conflicts of interest.

\section{Authors' Contributions}

Lele Li and Guoqing Yang contributed equally to this article.

\section{References}

[1] R. M. Chidiac and D. C. Aron, "Incidentalomas. A disease of modern technology," Endocrinology and Metabolism Clinics of North America, vol. 26, pp. 233-253, 1997.

[2] L. Barzon, N. Sonino, F. Fallo, G. Palu, and M. Boscaro, "Prevalence and natural history of adrenal incidentaloma," European Journal of Endocrinology, vol. 149, pp. 273-285, 2003. 
[3] P. K. Singh and H. N. Buch, "Adrenal incidentaloma: evaluation and management," Journal of Clinical Pathology, vol. 61, pp. 1168-1173, 2008.

[4] D. C. Aron, "The adrenal incidentaloma: disease of modern technology and public health problem," Reviews in Endocrine and Metabolic Disorders, vol. 2, pp. 335-342, 2001.

[5] M. Terzolo, A. Stigliano, I. Chiodini et al., "AME position statement on adrenal incidentaloma," European Journal of Endocrinology, vol. 164, pp. 851-870, 2011.

[6] J. Kim, K. H. Bae, Y. K. Choi et al., "Clinical characteristics for 348 patients with adrenal incidentaloma," Endocrinology and Metabolism (Seoul, Korea), vol. 28, pp. 20-25, 2013.

[7] X. Bin, Y. Qing, W. Linhui, G. Li, and S. Yinghao, "Adrenal incidentalomas: experience from a retrospective study in a Chinese population," Urologic Oncology, vol. 29, pp. 270-274, 2011.

[8] Y. Tabuchi, M. Otsuki, S. Kasayama et al., "Clinical and endocrinological characteristics of adrenal incidentaloma in Osaka region, Japan," Endocrine Journal, vol. 63, pp. 29-35, 2016.

[9] J. Patrova, I. Jarocka, H. Wahrenberg, and H. Falhammar, "Clinical outcome in adrenal incidentaloma: experience from one center," Endocrine Practice, vol. 21, pp. 870-877, 2015.

[10] F. Mantero, M. Terzolo, G. Arnaldi et al., "A survey on adrenal incidentaloma in Italy. Study group on adrenal tumors of the Italian Society of Endocrinology," The Journal of Clinical Endocrinology and Metabolism, vol. 85, pp. 637-644, 2000.

[11] A. Muth, L. Hammarstedt, M. Hellstrom et al., "Cohort study of patients with adrenal lesions discovered incidentally," The British Journal of Surgery, vol. 98, pp. 1383-1391, 2011.

[12] H. Ai-Thani, A. Ei-Menyar, M. Ai-Sulaiti et al., "Adrenal mass in patients who underwent abdominal computed tomography examination," North American Journal of Medical Sciences, vol. 7, pp. 212-219, 2016.

[13] S. Russi, H. T. Blument, and S. H. Gray, "Small adenomas of the adrenal cortex in hypertension and diabetes," Archives of Internal Medicine, vol. 76, pp. 284-291, 1945.

[14] R. R. Commons and C. P. Callaway, "Adenomas of the adrenal cortex," Archives of Internal Medicine, vol. 81, pp. 37-41, 1948.

[15] G. Arnaldi and M. Boscaro, "Adrenal incidentaloma," Best Practice \& Research. Clinical Endocrinology \& Metabolism, vol. 26, pp. 405-419, 2012.

[16] Y. Y. Cho, S. Suh, J. Y. Joung et al., "Clinical characteristics and follow-up of Korean patients with adrenal incidentalomas," The Korean Journal of Internal Medicine, vol. 28, pp. 557-564, 2013.

[17] H. Y. Kim, S. G. Kim, K. W. Lee et al., "Clinical study of adrenal incidentaloma in Korea," The Korean Journal of Internal Medicine, vol. 20, pp. 303-309, 2005.

[18] M. C. Yen, "Sonography of the adrenal glands: normal glands and small masses," American Journal of Roentgenology, vol. 135, pp. 1167-1177, 1980.

[19] E. Vassilatou, A. Vryonidou, D. Loannidis, S. A. Paschou, P. Maria, and I. Tzavara, "Bilateral adrenal incidentalomas differ from unilateral adrenal incidentalomas in subclinical cortisol hypersecretion but not in potential clinical implication," European Journal of Endocrinology, vol. 171, pp. 37-45, 2014.

[20] J. D. Pasternak, C. D. Seib, N. Seiser et al., "Differences between bilateral adrenal incidentalomas and unilateral lesions," JAMA Surgery, vol. 150, pp. 974-978, 2015.
[21] H. Olsen, E. Nordensrom, A. Bergenfelz, U. Nyman, S. Valdemarsson, and P. Erik, "Subclinical and CT appearance in adrenal incidentalomas: a multicenter study from Southern Sweden," Endocrine, vol. 42, pp. 164-173, 2012.

[22] V. Morelli, S. Palmieri, A. S. Salcuni et al., "Bilateral and unilateral adrenal incidentalomas: biochemical and clinical characteristics," European Journal of Endocrinology, vol. 168, pp. 235-241, 2013.

[23] S. Jaresch, E. Kornly, H. K. Kley, and R. Schlaghecke, "Adrenal incidentalomas and patients with homozygous or heterozygous congenital adrenal hyperplasia," The Journal of Clinical Endocrinology and Metabolism, vol. 74, pp. 685-689, 1992.

[24] H. Falhammar and D. J. Torpy, "Congenital adrenal hyperplasia due to 21-hydroxylase deficiency presenting as adrenal incidentaloma: a systematic review and meta-analysis," Endocrine Practice, vol. 22, pp. 736-752, 2016.

[25] G. Weijun, L. Lele, D. Jingtao et al., "Congenitaladrenal hyperplasia initially presenting with massive adrenal incidentalomas: a series of 4 cases," International Journal of Clinical and Experimental Medicine, vol. 9, pp. 13309-13318, 2016.

[26] M. Fassnacht, W. Arlt, I. Bancos et al., "Management of adrenal incidentalomas: European Society of Endocrinology Clinical Practice Guideline in collaboration with the European network for the study of adrenal tumors," European Journal of Endocrinology, vol. 175, pp. G1-G34, 2016.

[27] S. Y. Park, B. K. Park, J. J. Park, and C. Y. Kim, "CT sensitivities for large $(\geq 3 \mathrm{~cm})$ adrenal adenoma and cortical carcinoma," Abdominal Imaging, vol. 40, pp. 310-317, 2015. 


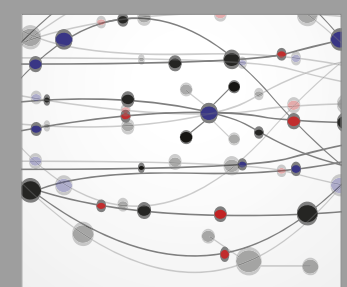

The Scientific World Journal
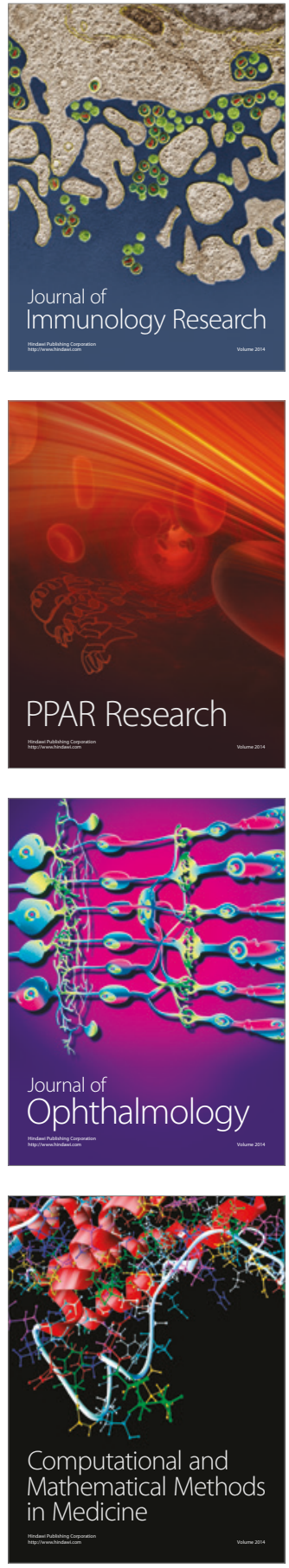

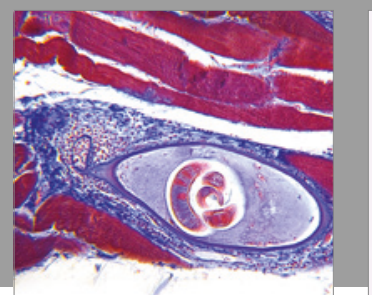

Gastroenterology Research and Practice
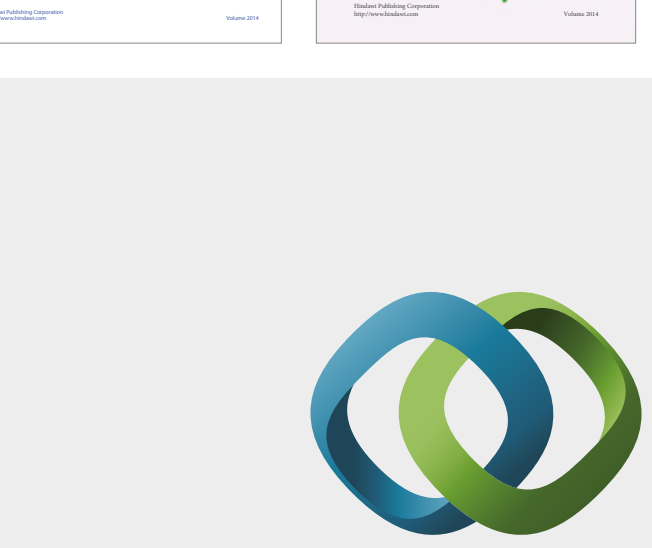

\section{Hindawi}

Submit your manuscripts at

https://www.hindawi.com
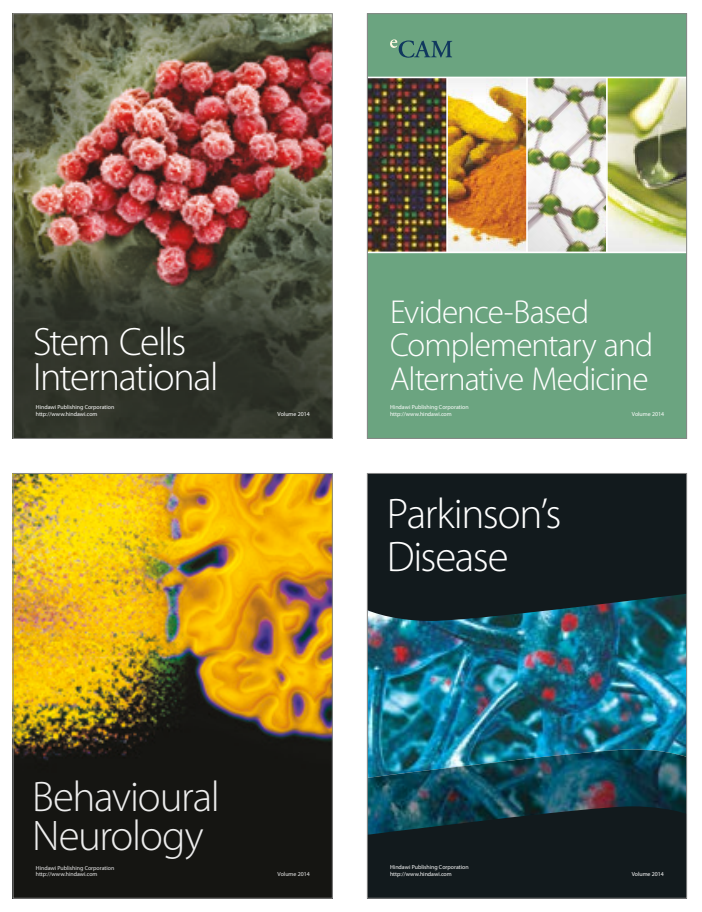
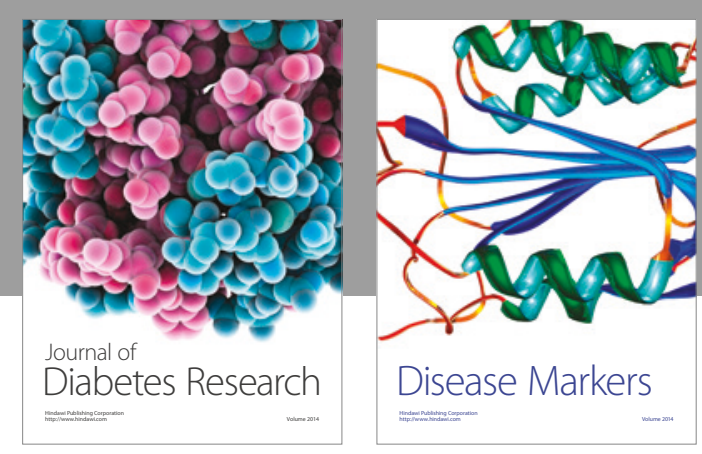

Disease Markers
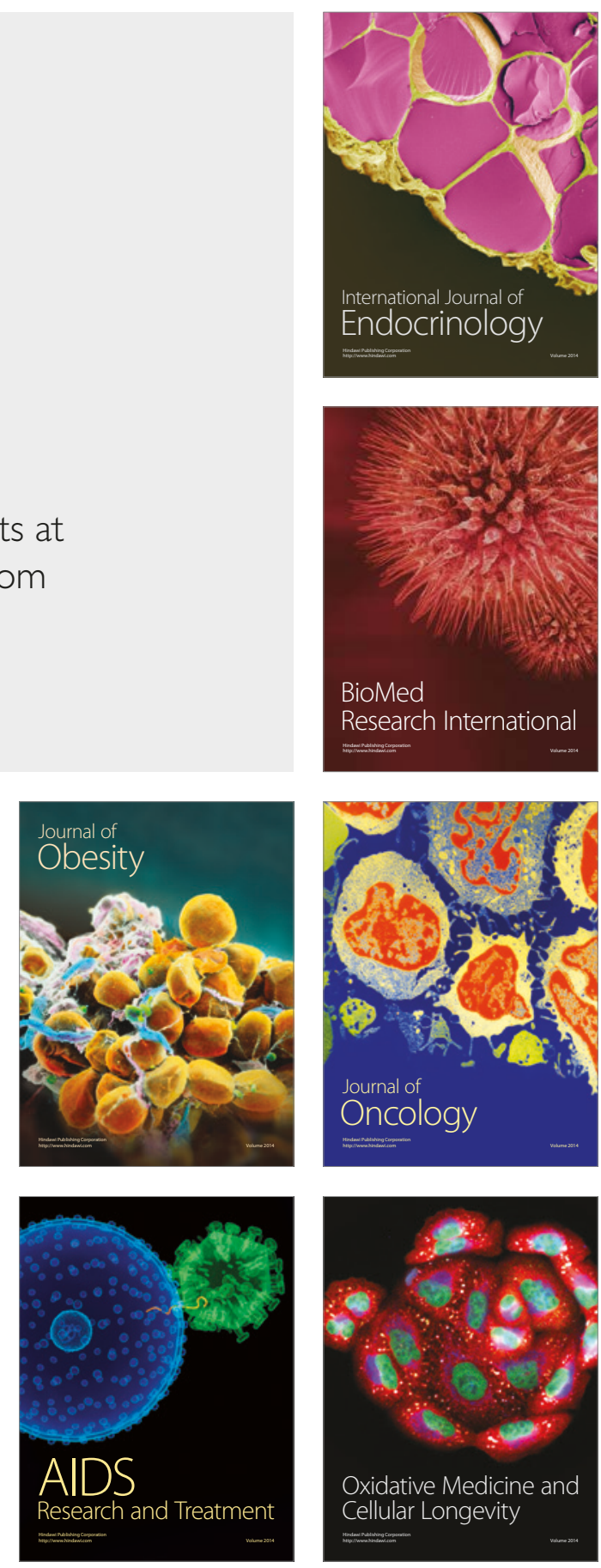